\title{
The Effect of Kinesio Tape on Factors for Neuromuscular Control of the Lower-Extremity: A Critically Appraised Topic
}

\author{
Nickolai Martonick, Kimber Kober, Abigail Watkins, Amanda DiEnno, Carmen Perez, Ashlie Renfro, \\ Songah Chae, and Russell Baker
}

\begin{abstract}
Clinical Scenario: Joint instability is a common condition that often stems from inadequate muscle activation and results in precarious movement patterns. When clinicians attempt to mechanically treat the unstable joint rather than attending to the underlying cause of the instability, patient outcomes may suffer. The use of kinesiology tape (KT) on an unstable joint has been proposed to aid in improving lower-extremity neuromuscular control. Clinical Question: Does KT improve factors of neuromuscular control in an athletic population when compared with no-tape or nonelastic taping techniques? Summary of Key Findings: The current literature was searched, and 5 randomized controlled studies were selected comparing the effects of KT with no-tape or nonelastic taping techniques on lower-extremity neuromuscular control in an athletic population. Primary findings suggest KT is not more effective than no-tape or nonelastic tape conditions at improving lower-extremity neuromuscular control in a healthy population. Clinical Bottom Line: The current evidence suggests that KT is ineffective for improving neuromuscular control at the ankle compared with nonelastic tape or no-tape conditions. KT was also found to be ineffective at improving hip and knee kinematics in healthy runners and cyclists. However, preliminary research has demonstrated improved neuromuscular control in a population displaying excessive knee valgus during a drop jump landing, after the application of KT. Clinicians should be cautious of these conflicting results and apply the best available evidence to their evaluation of the patient's status. Strength of Recommendation: There is grade B evidence that the use of KT on an athletic population does not improve biomechanical measures of ankle stability. There is inconclusive, grade B evidence that KT improves neuromuscular control at the knee in symptomatic populations.
\end{abstract}

Keywords: kinesiology tape, nonelastic tape, motor control, movement patterns

\section{Clinical Scenario}

Interventions used in sports medicine are often focused on addressing a symptom rather than the cause of the deficit or pathology. ${ }^{1}$ For example, the use of nonelastic athletic tape to reduce joint mobility and prevent injury is a typical symptom-focused intervention used in sport therapy. Instead of attempting to mechanically influence a perceived mechanical deficiency at the joint, it has been suggested that clinicians should identify why the joint is unstable, to determine if the cause is a local pathological condition or stems from regionally interdependent deficits. ${ }^{2}$ During movement, dynamic joint stability is achieved through both passive ligament restraints and muscle forces acting on the joint. ${ }^{1}$ Therefore, factors of neuromuscular control, such as muscle activation and strength, as well as the resulting kinetic and kinematic measures, should be considered when prescribing or assessing the effectiveness of an intervention.

A common cause of injury or increased joint mobility is insufficient neuromuscular control, which has been found to increase the risk of various lower-extremity injuries in an athletic population. ${ }^{1}$ Multiple investigations have demonstrated that increased knee abduction (knee valgus) is correlated with a greater risk of anterior cruciate ligament injuries in female athletes. ${ }^{1,3}$ The mechanism behind this relationship is thought to arise from the inability of neuromuscular control to dissipate forces at the knee during high load movements. ${ }^{1}$ Dynamic knee valgus (DKV) is a practical example of an at-risk movement pattern, thought to arise

The authors are with the University of Idaho, Moscow, ID, USA. Martonick (nmartonick@uidaho.edu) is corresponding author. from insufficient neuromuscular control of the hip girdle. ${ }^{3}$ Though there are many factors for noncontact anterior cruciate ligament injury, an intervention focused on improving precarious movement patterns through improved neuromuscular control may be the most effective method for decreasing the risk of injury. ${ }^{1,3}$

Elastic tape applications, commonly referred to as kinesiology tape (KT), have become popular interventions among rehabilitation professionals in recent years. An increased prevalence may be attributed to KT brands claiming the tape can be used for neuromuscular reeducation. ${ }^{4}$ Given the increased popularity of KT in clinical settings, there is a need to determine if a KT application has a positive or negative effect on neuromuscular control. Exploring the use of KT applications compared with other nonelastic tape applications or no-tape applications is necessary to better understand whether KT applications improve neuromuscular control and have a place in clinical practice.

\section{Focused Clinical Question}

Does KT improve factors of neuromuscular control in an athletic population when compared with no-tape or nonelastic taping techniques?

\section{Summary of Search, "Best Evidence" Appraised, and Key Findings}

- The literature was searched to identify peer-reviewed articles that investigated the comparison of KT with no-tape or nonelastic tape conditions in athletes. 
- Of the 21 identified articles, 4 were randomized crossover studies and 1 article was a randomized controlled trial.

- Seven articles were excluded for using participants with current injuries or pain, 6 articles were published before 2010, 3 articles included glenohumeral motion, and 1 article included a secondary school population.

- An application of KT reduced knee valgus in males and females, and the improved movement patterns were maintained in the female group 3 days following application. ${ }^{2}$

- No significant differences in ankle joint proprioception were found between KT and no-tape conditions. ${ }^{2,5}$

- Electromyography measures of rectus femoris to biceps femoris muscle ratio were demonstrated to significantly decrease in professional cyclists, following a KT application.

\section{Clinical Bottom Line}

There is minimal evidence to support the use of KT for improved lower-extremity motor control in a healthy athletic population when compared with no intervention. Current evidence is inconclusive across multiple populations and taping applications, rendering it difficult for clinicians to determine an appropriate use for KT. However, when a KT application was administered to a population with precarious movement patterns, short-term benefits were observed. Consideration for the use of KT in patient care must include diagnostic expertise, a patient's needs, and the clinical context. Clinicians should also be mindful of the potential for taping applications to alter biomechanical behavior away from the site of application, as demonstrated by 2 studies reviewed in this article. Further research is needed to determine the biomechanical effects of KT tape applications directed at improving the movement patterns of populations that are thought to be at risk for injury.

\section{Strength of Recommendation}

The strength of these recommendations is supported using the Strength of Recommendation Taxonomy. ${ }^{6}$ Grade A evidence indicates that the recommendation is based on consistent, goodquality evidence. ${ }^{6}$ Grade B evidence indicates that the recommendation is based on inconsistent or limited-quality evidence. ${ }^{6}$ Grade $\mathrm{C}$ evidence indicates that the recommendation is based on consensus, usual practice, or expert opinion. ${ }^{6}$ There is Grade B evidence that the use of KT is ineffective for improving lower-extremity neuromuscular control at the ankle. At the knee, there is grade B evidence to support the use of KT compared with no tape (NT) or Mulligan Concept taping (MCT). There is grade B evidence to support the use of MCT for reducing kinetic forces at the hip and knee. There is grade B evidence that traditional nonelastic ankle taping is more effective than KT and NT at improving neuromuscular control of the ankle.

\section{Search Strategy}

\section{Terms Used to Guide Search Strategy}

- Patient/Client Group: athletic population

- Intervention/Assessment: KT and kinesio tape

- Comparison: no tape and nonelastic tape

- Outcomes: lower-extremity neuromuscular control, electromyography, kinematics, and kinetics

\section{Sources of Evidence Searched}

- MEDLINE

- LILI

- ScienceDirect

- Additional references obtained via reference list review and hand search

\section{Inclusion Criteria}

- Adult, athletic population

- At least one outcome measuring factors of neuromuscular control

- Articles published after 2010

- Studies classified as level 2 evidence or higher on the Oxford Center for Evidence-Based Medicine (CEBM) Scale ${ }^{8}$

\section{Exclusion Criteria}

- Participants with orthopedic injuries

- Studies of upper-extremity neuromuscular control

- Studies examining the effect of KT on pain

- KT not included as a treatment condition

- Articles not published in English

\section{Results of Search}

For this study, 21 articles were identified, with 5 articles meeting the inclusion criteria (Table 1). The level of evidence was assessed using the Template for Intervention Description and Replication (TIDieR) checklist and the Oxford CEBM ${ }^{8}$ In addition, each article was scored using the PEDro scale to assess the validity of the studies following standard scoring procedures.

\section{Best Evidence}

As described in Table 2, the studies selected for inclusion in this critically appraised topic were identified as the best evidence. The authors of these level 1 and level 2 studies considered the use of KT for targeting joint kinematics, kinetics, muscle activation, and center of pressure (COP) in comparison with nonelastic tape or no-tape conditions.

\section{Implications for Practice, Education, and Future Research}

Traditionally, sports therapists have utilized a nonelastic tape intended to mechanically restrict excessive range of motion (eg, sudden ankle perturbation) through braces and nonelastic tape applications. ${ }^{2}$ However, investigators have begun to examine the hypothesized effects of elastic tape on the stimulation of mechanoreceptors through skin stretch to facilitate proprioception; thus, improving neuromuscular control and ultimately, an athlete's movement patterns. ${ }^{2}$ To examine KT as a potential intervention for athletes at risk for ankle trauma, investigators analyzed the COP of professional soccer players when comparing a KT application with a no-tape condition. ${ }^{4}$ Though there is no gold standard for measuring ankle proprioception, the investigators utilized a moving target program from the balance module of a Kin-Com ${ }^{\circledR} 125 \mathrm{AP}$ isokinetic dynamometer (East Ridge, TN). The participants controlled their 
Table 1 Levels of Evidence Selected Using the Oxford CEBM Guidelines ${ }^{7}$

\begin{tabular}{llcc}
\hline Level of evidence & Study design/methodology of article retrieved & Number located & Study \\
\hline Level $1 \mathrm{~b}$ & Double-blinded, randomized controlled study & 1 & Rajasekar et al $^{3}$ \\
Level $1 \mathrm{~b}$ & Single-blind randomized crossover study & 3 & Bailey and Firth $^{4}$ \\
& & Howe et al $^{9}$ & Briem et al $^{2}$ \\
Level $2 \mathrm{~b}$ & Randomized crossover study & 1 & Hebert-Losier et al $^{5}$ \\
\hline
\end{tabular}

COP to track an on-screen visual stimulus to simulate the ankle instability that might occur in a soccer match. ${ }^{4}$ Researchers observed that an application of KT had no significant effect on the COP of professional soccer players. ${ }^{4}$ Although no positive effect was observed following the application of KT, consideration should be given to the population in question, as this finding may not translate to amateur athletes or the general population.

Although the previous study examined the potential of KT to increase ankle stability through improved proprioception, KT has also been proposed to improve muscle activation. ${ }^{2}$ Therefore, investigators examined whether KT was effective as a preventative measure for ankle trauma by using surface electromyography to assess the effectiveness of KT at facilitating the muscle activation of fibularis longus. ${ }^{2}$ The participants stabilized on a balance board with the tested limb as the board was subjected to perturbation from a $10-\mathrm{kg}$ weight. ${ }^{2}$ Muscle activity was assessed at 3 time points for mean, peak, and time to peak electromyography data. ${ }^{2}$ When compared with a nonelastic tape and no-tape condition, KT was not found to be effective for improving fibularis muscle activation (thought to be impaired in those with functional ankle instability) during sudden ankle perturbation. ${ }^{2}$ Furthermore, Briem et $\mathrm{al}^{2}$ demonstrated a greater increase in fibularis longus activation from the nonelastic tape group, suggesting that a tape that creates more pull on the skin (ie, nonelastic tape) may better increase mechanoreceptor stimulation. ${ }^{2}$ However, clinicians should be cautious of laboratory experiments utilizing a nonelastic white tape because it has been demonstrated to decrease in its tensile strength during exercise, potentially reducing the ability of the tape to cue muscle activity throughout the course of athletic competitions of longer duration. ${ }^{2}$

The use of KT has also been proposed as an intervention option to manage and prevent knee injuries associated with biomechanical deficiencies. ${ }^{8,9}$ Traditional bracing of the knee to prevent injury during athletic movements is cumbersome and may not address all potential drivers of inefficient biomechanics. ${ }^{8}$ Elastic tapes, as well as other tape applications, have been hypothesized as an alternative to improve joint kinematics and reduce the risk factors for knee trauma. ${ }^{8}$ For example, researchers hypothesized that an application of KT utilized to facilitate the activation of the gluteus medius could improve $\mathrm{DKV}$, which has been demonstrated as a risk factor for anterior cruciate ligament ruptures. ${ }^{3}$ When tested acutely, DKV, as well as the muscle strength of the gluteus medius (as measured by the Donetelli Leg Drop Test), was reported to have improved after the application of KT. ${ }^{3}$ The participants in this study presented without injury, but were required to demonstrate impaired motor control through the presence of DKV $\left(>8^{\circ}\right.$ for men and $>13^{\circ}$ for women) during a drop jump. ${ }^{3}$ Unlike the other 4 studies presented, Rajasekar et $\mathrm{al}^{3}$ studied the effects of KT on participants with known impairments in lower-extremity movement patterns and found significant improvements following the application of KT. Though results from this study are promising for clinicians looking to improve at-risk movement patterns, 3D motion capture analysis might have provided more insight into the kinematics of the hip, knee, and ankle in all planes of motion.

Recently, researchers compared a KT application with an MCT application and no application of tape to assess the potential biomechanical changes from different tape applications targeted at improving patella femoral pain syndrome. KT was applied in a manner to facilitate a medial glide of the patella and potentially improved activation of the vastus medialis oblique. ${ }^{9}$ The MCT application is postulated to improve patella tracking through a mechanical internal rotation of the tibia. ${ }^{9}$ Participants performed $10-\mathrm{m}$ "run throughs" while being analyzed by $3 \mathrm{D}$ motion capture cameras and force plates. 9 When compared with the no-tape condition, healthy runners with an application of KT and MCT demonstrated no significant differences from their baseline data for hip and knee angles. However, when MCT was applied, healthy runners were found to have decreased knee and hip extensor moments when compared with KT. ${ }^{9}$ The results of this study do not demonstrate that a KT application intended for patients with patella femoral pain syndrome will alter the biomechanics of a healthy population. However, clinicians should be attentive to the potential for tape to alter biomechanics at segments of the kinetic chain away from the sight of application, as demonstrated by the MC application.

Hebert-Losier et $\mathrm{al}^{5}$ investigated the effect of KT compared with a no-tape application on knee kinematics in professional cyclers. The investigators hypothesized that an increase in muscle activation would result in changes to knee kinematic values during cycling. ${ }^{5}$ Participants completed 4 minutes of cycling at 100 and $200 \mathrm{~W}$, while 3D motion capture cameras and surface electromyography captured data values under KT and no-tape conditions. ${ }^{5}$ When compared with the no-tape condition, cyclers with KT demonstrated no difference in lower-extremity kinematic values. ${ }^{5}$ The investigators did find a significant increase in vastus medialis activity, as well as a decrease in the ratio of rectus femoris to biceps femoris activation following a simple application of KT across the patella. ${ }^{5}$ The demonstrated improvements in the coactivation of biceps femoris and rectus femoris may benefit individuals with quad-dominant movement strategies. ${ }^{5}$ However, further investigation into the effect of this specific taping application on a population with movement strategies considered to be quad-dominant (ie, DKV) would provide more applicable evidence for the guidance of clinical practice.

In conclusion, a consensus on the effectiveness of KT could not be reached from the 5 studies examined in this critically appraised topic. Although the evidence to support the use of KT to improve factors regarding neuromuscular control is insufficient, the strength of these recommendations is limited due to a lack of consistency between study methods, as well as transferability to practice. Not only did applications of KT vary, but motor patterns performed by participants varied as well. For these reasons, clinical implications must be extrapolated on a study-by-study basis at this time, until a stronger, more homogenous knowledge base has been established to 


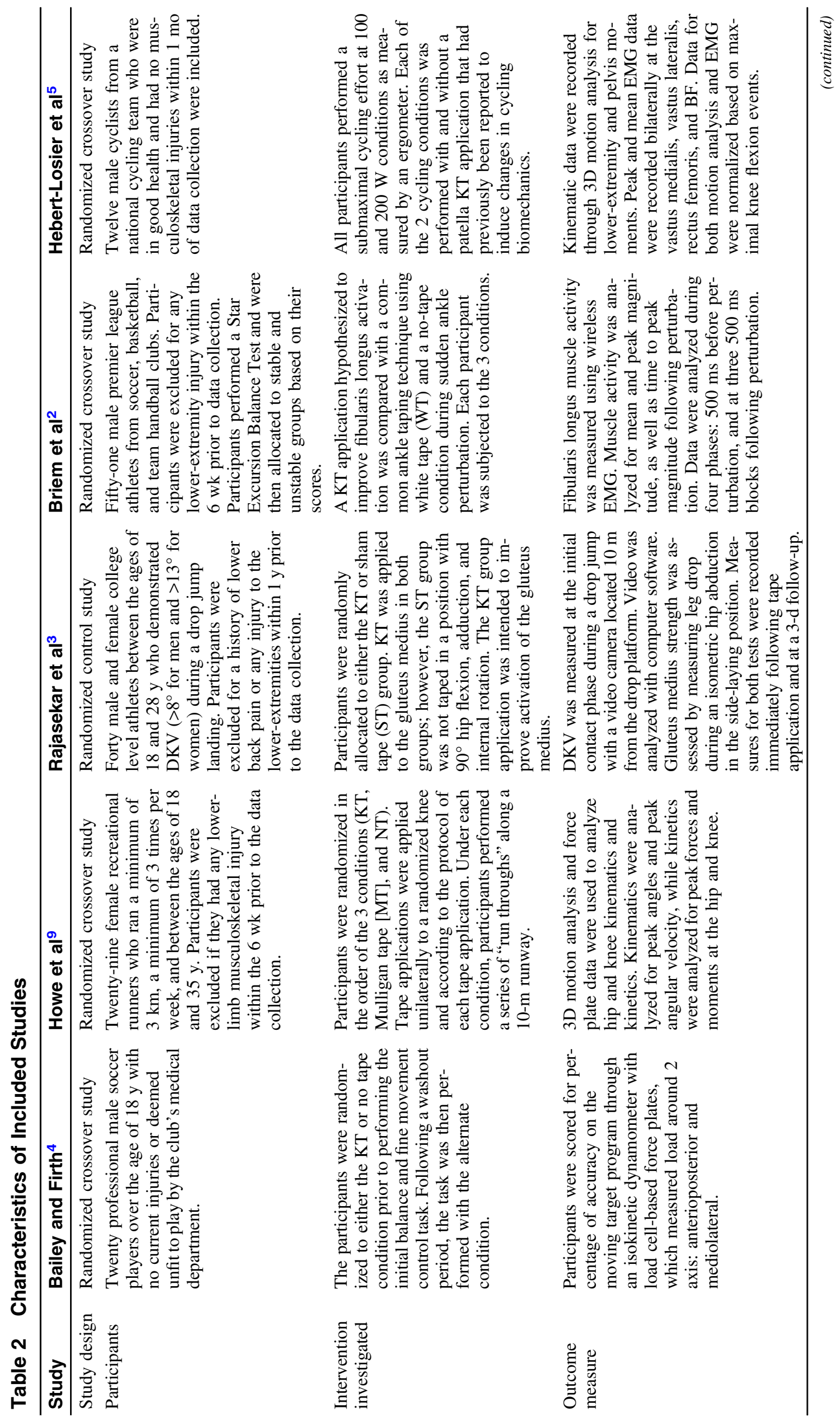




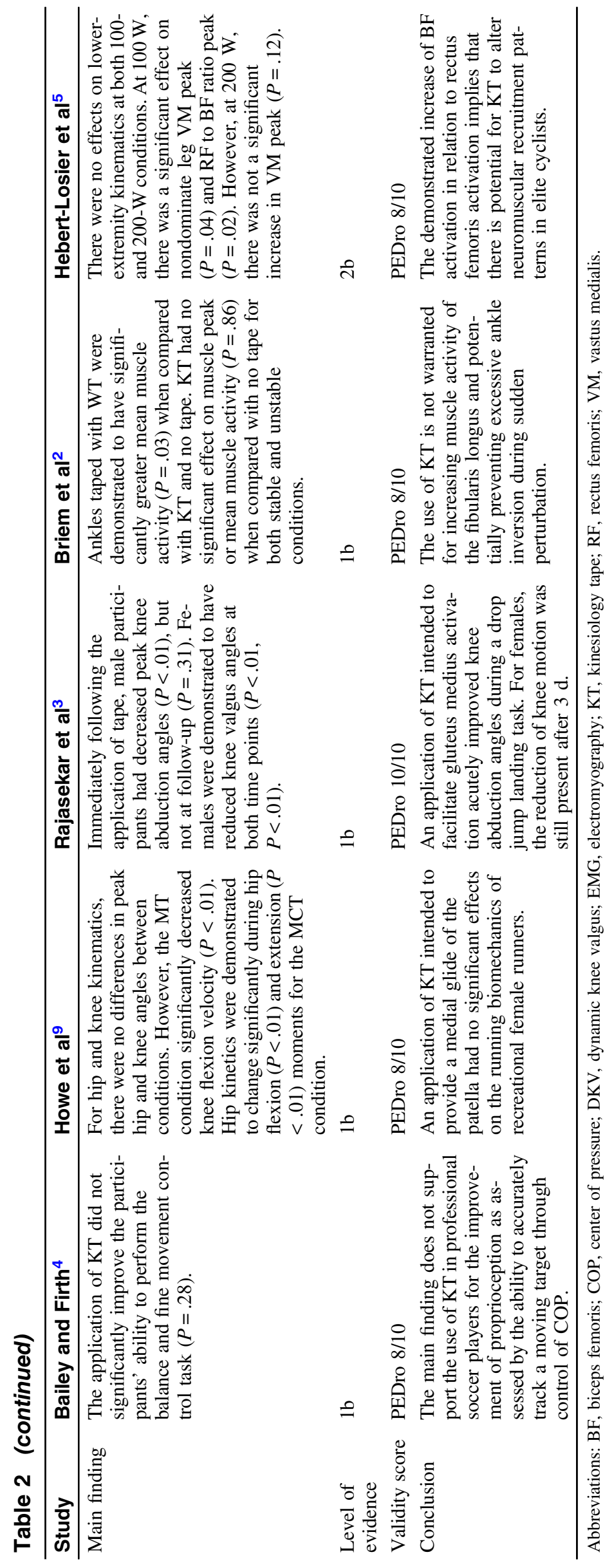


evaluate KT in comparison with other taping materials and methods. To better solidify current knowledge, future research should address the existing evidence with more consistent study designs and further address the potential for KT applications to improve the neuromuscular control and movement patterns of populations displaying excessive joint movement. This critically appraised topic should be reassessed in 2 years to determine if additional evidence supports $\mathrm{KT}$ as an intervention to improve lower-extremity motor control and prevent injury in an athletic population.

\section{Acknowledgments}

The authors of this study would like to acknowledge the university faculty who instructed the authors through the process of scholarship. There are no conflicts of interest to report.

\section{References}

1. Hewett TE, Myer GD, Ford K, et al. Biomechanical measures of neuromuscular control and valgus loading of the knee predict anterior cruciate ligament injury risk in female athletes. Am J Sports Med. 2009;33(4):492-501. doi:10.1177/0363546504269591

2. Briem K, Eythörsdöttir H, Magnúsdóttir RG, Pálmarsson R, Rúnarsdöttir T, Sveinsson T. Effects of kinesio tape compared with nonelastic sports tape and the untaped ankle during a sudden inversion perturbation in male athletes. J Orthop Sports Phys Ther. 2011;41(8): 328-335. doi:10.2519/jospt.2011.3501
3. Rajasekar S, Kumar A, Patel J, Ramprasad M, Samuel AJ. Does kinesio taping correct exaggerated dynamic knee valgus? A randomized double blinded sham-controlled trial. J Bodyw Mov Ther. 2018;22(3):727-732. doi:10.1016/j.jbmt.2017.09.003

4. Bailey D, Firth P. Does kinesiology taping of the ankles affect proprioceptive control in professional football (soccer) players? J Orthop Sports Phys Ther. 2017;25:94-98.

5. Hébert-Losier K, Yin NS, Beaven CM, Tee CCL, Richards J. Physiological, kinematic, and electromyographic responses to kinesiology-type patella tape in elite cyclists. J Electromygr Kinesiol. 2019;44:36-45. doi:10.1016/j.jelekin.2018.11.009

6. Ebell MH, Siwek J, Weiss BD, et al. Strength of recommendation taxonomy (SORT): a patient-centered approach to grading evidence in the medical literature. J Am Board Fam Pract. 2004;17(1):59-67. PubMed ID: 15014055 doi:10.3122/jabfm.17.1.59

7. Howick J, Chalmers I, Glasziou P, et al. The oxford 2011 level of evidence. Oxford Centre for Evidence-Based Medicine. 2011. http:// www.cebm.net/index.aspx?o=5653. Accessed May 10, 2019.

8. Hanzlikova I, Richars J, Tomsa M, et al. The effect of proprioceptive knee bracing on knee stability during three different sport related movement tasks in healthy subjects and the implications to the management of Anterior Cruciate Ligament (ACL) injuries. Gait Posture. 2016;48:165-170. PubMed ID: 27267771 doi:10.1016/j. gaitpost.2016.05.011

9. Howe A, Campbell A, Hall T, Hopper D. Effects of two different knee tape procedures on lower-limb kinematics and kinetics in recreational runners. Scand J Med Sci Sports. 2015;25:517-524. doi:10.1111/sms.12269 